Table S5: Pseudocode for the regEFMtool. Text sections in italic style indicate our modifications compared to the binary method reported by Gagneur and Klamt [5].

\title{
Pre-processing phase
}

Read input files (stoichiometric matrix, reversibility, ...)

Split each reversible reaction into two irreversible reactions

Read file containing gene regulatory information

Parse gene regulation file

LOOP: Iterate through all provided rules

Create reverse polish notation (RPN) stack for rule

Add RPN stack to post-processing rule container

IF rule qualifies for iteration phase THEN

Create bit pattern for rule

Add bit pattern to iteration phase rule container

END IF

END OF LOOP

Compute kernel $\mathrm{K}$ of stoichiometric matrix

Use kernel $\mathrm{K}$ to initialize mode matrix $\mathrm{R}$

Sort mode matrix $\mathrm{R}$ (put rows with only positive elements to top)

Convert all rows with only positive elements to binary format

\section{Iteration phase}

LOOP1: Iterate through all numerical rows of mode matrix $\mathrm{R}$

LOOP2: Iterate through all adjacent positive-negative pairs

Create new mode by combining parent modes

LOOP3: Iterate through all iteration phase rules

IF mode does not obey rule THEN

Eliminate mode

LAST LOOP 3

END IF

END OF LOOP3

END OF LOOP2

Remove all modes with negative values at current row

Convert current row to binary format

END OF LOOP1

\section{Post-processing phase}

LOOP1: Iterate through all modes

LOOP2: Iterate through all post-processing rules

IF mode does not obey rule THEN

Remove mode

LAST LOOP2

END IF

END OF LOOP2

END OF LOOP1

Remove futile 2-cycle modes

Convert binary modes to numerical form

Write result file 